\title{
Triply-resonant Optical Parametric Oscillator by Four-wave Mixing with Rubidium Vapor inside an Optical Cavity
}

\author{
Xudong $\mathrm{Yu}^{1}$, Min $\mathrm{Xiao}^{2}$, and Jing Zhang ${ }^{1 \dagger}$ \\ ${ }^{1}$ The State Key Laboratory of Quantum Optics Quantum Optics Devices, \\ Institute of Opto-Electronics, Shanxi University, Taiyuan 030006, P.R. China and \\ 2 Department of Physics, University of Arkansas, Fayetteville, Arkansas 72701, USA
}

\begin{abstract}
We present an experimental demonstration of simultaneous above-threshold oscillations of the Stokes and anti-Stokes fields together with the single pumping beam with rubidium atoms inside an optical standing-wave cavity. The triple resonant conditions can be achieved easily by making use of the large dispersions due to two-photon transitions in the three-level atomic system. This work provides a way to achieve high efficient nonlinear frequency conversion and the generated bright Stokes and anti-Stokes cavity output beams are potential resource for applications in quantum information science.
\end{abstract}

Various atomic systems have been used as intracavity gain media to realize cavity oscillations [1 7$]$. Especially, multi-level atomic systems have more interesting characteristics and can be more efficient in managing the absorption and dispersion properties of the intracavity gain medium for building up resonance simultaneously of different cavity modes. It is easy to have an off-resonant pump beam to make the Stokes or anti-Stakes field be on resonance with one of the cavity modes in a three-level $\Lambda$-type atomic system, and get it to oscillate with a large pump power [7]. When the pump beam is tuned near one of the atomic transitions in a three-level $\Lambda$ system inside an optical cavity, cavity field oscillation (or lasing without population inversion) can occur in the frequency corresponding to the other atomic transition [8]. This system can be considered as an optical parametric oscillator (OPO) since the atomic variables can be adiabatically eliminated in treating the atom-field interactions [9, 10].

In this Letter, we present our experimental demonstration of a triply-resonant atom-cavity system, as shown in Fig.1. With one pump laser beam (frequency $\omega_{p}$ ) tuned to the cavity resonance as the cavity input, two optical fields, both the Stokes field (frequency $\omega_{b}$ ) and anti-Stokes field (frequency $\omega_{a}$ ), are generated simultaneously. The frequency of the pump beam is detuned from the atomic resonances $|1\rangle \leftrightarrow|0\rangle$ and $|2\rangle \leftrightarrow|0\rangle$ by the amount of $\Delta_{b}=\omega_{p}-\omega_{01}$ and $\Delta_{a}=\omega_{p}-\omega_{02}$ (thus $\Delta_{a}-\Delta_{b}=\omega_{12}$ ), which generates two sidebands at frequencies of $\pm \omega_{12}$ from the pump beam frequency, respectively. Typically, the generated Stokes and antiStokes fields are difficult to be made on resonance with the cavity modes at the same time, since the cavity mode has already been tuned to be on resonance with the pump field. However, since we work with the naturally mixed ${ }^{87} R b$ and ${ }^{85} R b$ vapor cell as the intracavity medium, there are several broad absorption bands in the transmission spectrum, as shown in Fig. 2(a). The weak field cavity transmission spectrum is given in Fig. 2(b), which shows the cavity transmission peaks with modified peak separations caused by the enhanced dispersions associated

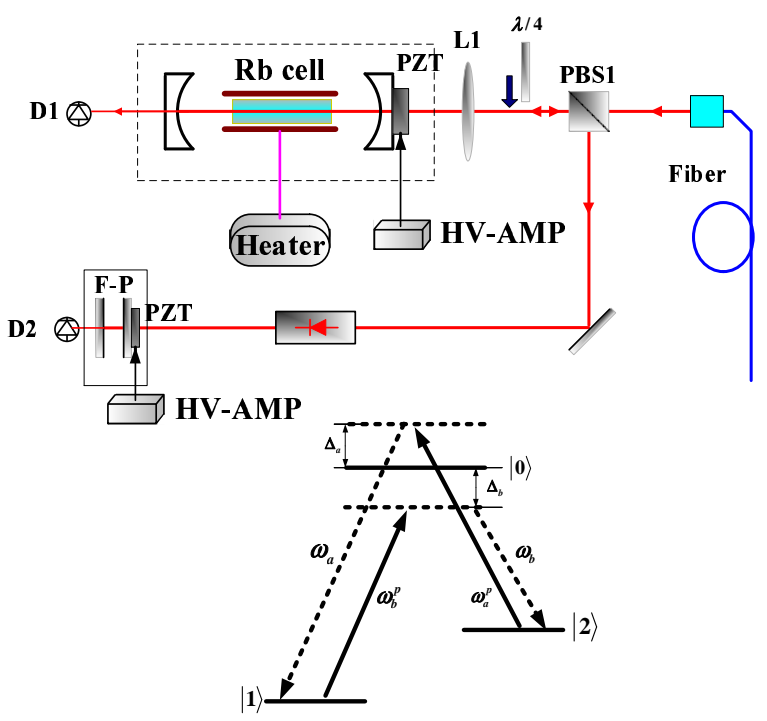

FIG. 1: (Color online). Schematic of the experimental setup of the coupled three-level atoms-cavity system. $\lambda / 4$ : quarterwave plate; D1, D2: detectors; HV-AMP: high voltage amplifier; PZT: piezoelectric transducer; PBS: polarized beam splitter; L: optical lens; F-P: Febry-Perot cavity. Bottom: relevant energy levels and field configurations. $\omega_{a}^{p}=\omega_{b}^{p}=\omega_{p}$ is the frequency of the single pump field.

with the tails of the absorption lineshape at high atomic density [11]. By manipulating the large intracavity dispersions via pump laser frequency detuning, the Stokes and anti-Stokes fields can be made to be simultaneously on resonance with the modified cavity modes together with the pump field, which is very similar to the case of a triple-resonant OPO with nonlinear crystals [12 19].

The experiment is done by placing a naturally mixed rubidium vapor cell inside an optical standing-wave cavity of length $17.7 \mathrm{~cm}$. The cavity is composed of two curved mirrors with the same radius of curvature of 100 $\mathrm{mm}$. The reflectivity is $90 \%$ at $780 \mathrm{~nm}$ for the input coupler $M 1$ (on right), which is mounted on a PZT to adjust the cavity length. The left mirror $M 2$ has a reflectivity of 
$99.5 \%$ at $780 \mathrm{~nm}$. The finesse of the cavity (including the losses of two faces of the atomic cell) is about $F=20$. The length of the vapor cell is $7.5 \mathrm{~cm}$. Thus we may obtain that the cavity bandwidth (the half-width at half maximum for the cavity) is about $21 \mathrm{MHz}$ and the OPO efficiency (output coupling over total losses) about $20 \%$. The temperature of the vapor cell can be controlled by a heater. A beam from a grating-stabilized diode laser is injected into a tapered amplifier (TA). The high power output from the TA then passes a standard polarization maintaining single-mode fiber, which is used as the cavity pump beam with an input power of $100 \mathrm{~mW}$. The pump laser beam with the spatial mode filter by the optical fiber is easy to be mode-matched to the $T E M_{00}$ mode of the optical cavity. This atom-cavity system is studied by monitoring the cavity reflection spectra using a scanned F-P cavity. We explore two different input and output configurations of the cavity to detect the different polarizations of the generated Stokes and anti-Stokes fields. One is that the pump field first passes through a polarized beamsplitter (PBS) and is injected into the cavity with horizontal polarization. The vertically-polarized component of the cavity reflection field is reflected by the same PBS, which mainly contains the generated Stokes and anti-Stokes fields with only a little pump field. The total reflected field then passes through an optical isolator and is monitored by a scanned F-P cavity. The other configuration is to use the pump field to inject into the cavity with circular polarization by passing through a $\lambda / 4$ waveplate. The output pump field from cavity reflection is reflected by the PBS when passes through the $\lambda / 4$ waveplate again. Thus, the total reflected field from the PBS contains the reflected pump field from the cavity and also the generated Stokes and anti-Stokes fields with the same polarization as the pump field inside the cavity.

Figure 3 presents the F-P cavity transmission spectra when the pump beam frequency is set at different positions as indicated in Fig. 2(a) and the pump, Stokes and anti-Stokes fields build up on resonance in the cavity simultaneously. First, as the pump frequency $\omega_{p}$ is tuned to the frequency as indicated by the arrow $a$, the F-P cavity transmission spectrum is given by Fig. 3(a). The pump beam power is set at $100 \mathrm{~mW}$. The two nearby side peaks (separated from the large middle peak of the pump light by $\pm 6.8 \mathrm{GHz}$ ) are from the generated Stokes and anti-Stokes fields of the ${ }^{87} R b$ atoms, which oscillate above thresholds. The central peak and the free-spectral range (FSR) are for the pump beam. The different peak heights (corresponding to the output power) for the Stokes and anti-Stokes fields come from several mechanisms including the cavity detuning, the dependence of gain on the detuning of the pump field, and the variation of intracavity absorption by other atomic transitions (as can be easily seen from Fig. 2). The two outer small peaks on both sides are also the Stokes and anti-Stokes fields due

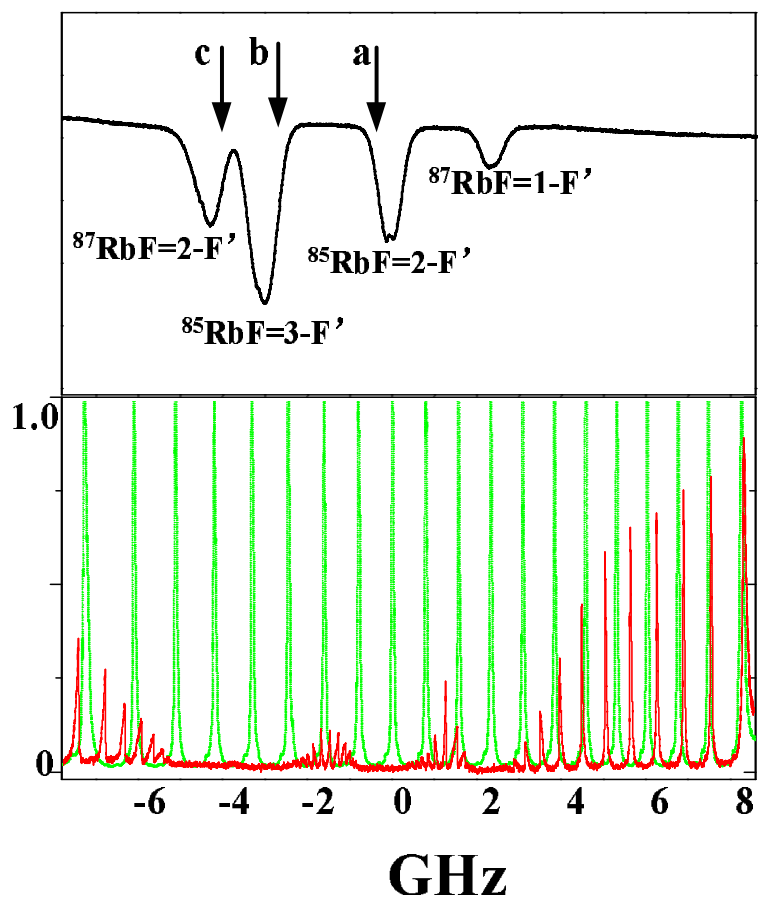

FIG. 2: (a) The saturated absorption spectrum is shown for the frequency reference of the pump light. (b) The weak-field cavity transmission spectrum with atoms inside the cavity (red line). The empty cavity transmission spectrum without atoms (green line).

to the periodicity of the scanned F-P cavity (i.e. FSR). As the pump frequency is tuned to the position marked by arrow $b$, the F-P cavity transmission spectrum given by Fig. 3(b) presents the generated Stokes field to be on resonance with the cavity and above threshold, where the three-level atomic system is ${ }^{85} R b$ atoms with the ground hyperfine state separation of about $3.035 \mathrm{GHz}$. The antiStokes field is absorbed inside the cavity below threshold. When the pump frequency is further tuned to the red side (arrow c), both F-P cavity transmission peaks for the Stokes and anti-Stokes fields appear simultaneously, showing triple-resonant oscillations for the atom-cavity system. Under both different polarization configurations of the cavity input field, we can detect the generated Stokes and anti-Stokes fields with different polarizations above thresholds.

The measurement of the oscillation threshold for the anti-Stokes field (shown in Fig. 3(a)) is presented in Fig.4. The cavity oscillation starts gradually at low input pump power, and saturates at high pump power as expected in a laser-like system. The threshold behaviors for the Stokes and anti-Stokes fields in other cases (for examples Figs. 3(b) and (c)) are similar. The total output powers of the Stokes and anti-Stokes fields can reach more than $1 \mathrm{~mW}$ when the pump power is about 100 $m W$, which indicates that such OPO system with multi- 


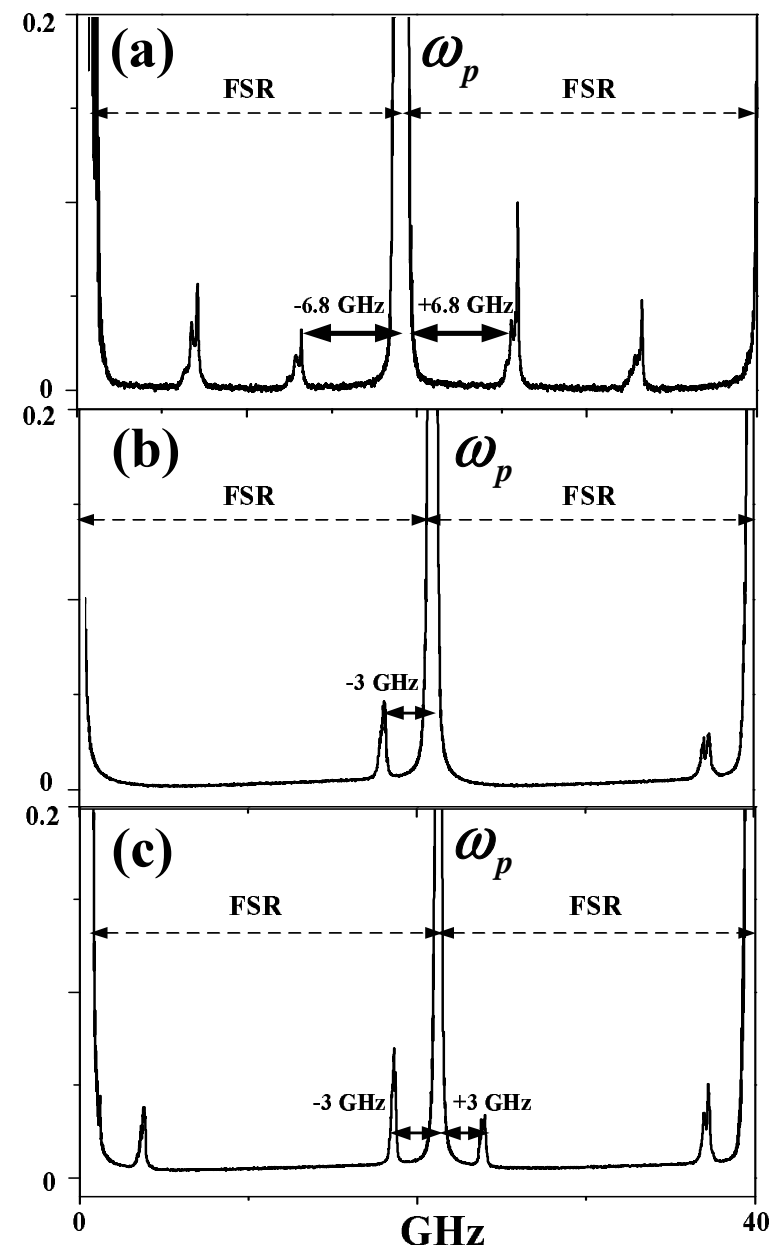

FIG. 3: The scanned F-P cavity transmission spectra. (a), (b) and (c) show the generated Stokes and anti-Stokes fields when the pump frequency is set at different positions $a, b$, and $\mathrm{c}$ respectively as indicated in Fig. 2(a). The temperature of the vapor cell is set at $105^{\circ} \mathrm{C}$.

level atoms can be very efficient. The current double- $\Lambda$ atomic configuration is an ideal system to generate correlated Stokes and anti-Stokes photon pairs [20]. With such atomic medium inside an optical cavity and driven above oscillation thresholds, bright correlated twin beams can be obtained, similar to the triple-resonant nondegenerate OPOs above thresholds with $\chi^{(2)}$ nonlinear crystal [12 19]. The bright Stokes and anti-Stokes cavity output beams above threshold will possess the different quantum characteristics comparing with that produced from single-pass four-wave mixing process with coherent signal injection [20]. Many interesting phenomena can be realized using this demonstrated triple-resonant OPO system in double- $\Lambda$ atomic system, especially with easily managed large intracavity dispersions.

In conclusion, we have experimentally demonstrated simultaneous resonances of the generated Stokes and antiStokes fields, together with the single pump field, in an

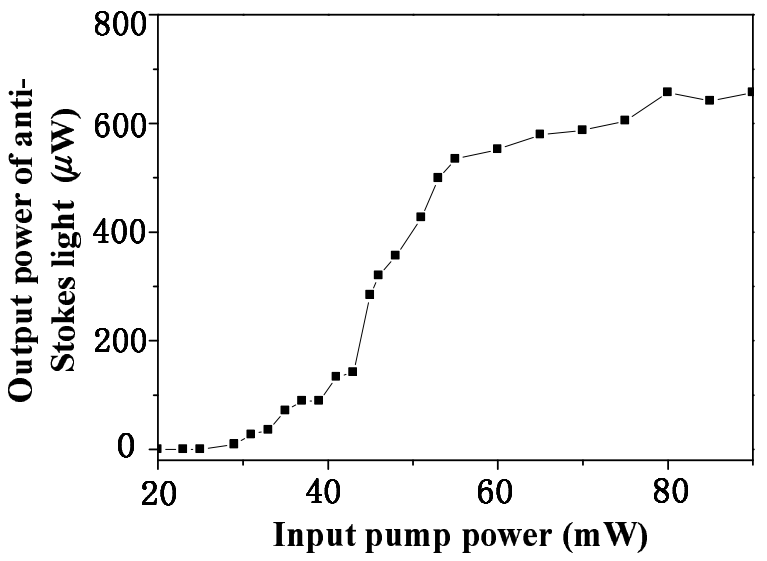

FIG. 4: The measured threshold behavior of the emitted antiStokes light in Fig. 3(b) as a function the pump beam power. The temperature of the $\mathrm{Rb}$ cell is $T=105^{\circ} \mathrm{C}$.

optical cavity. The modified large dispersions due to the intracavity dense atomic medium are very important in allowing such triple-resonant conditions to be simultaneously satisfied. Such experimental system can be useful to study atom-cavity interaction, especially for quantum entangled beams in quantum information processing [21].

${ }^{\dagger}$ Corresponding author's email address: jzhang74@yahoo.com, jzhang74@sxu.edu.cn

We thanks K. Peng, C. Xie and T. Zhang for the helpful discussions. This research was supported in part by NSFC for Distinguished Young Scholars (Grant No. 10725416), National Basic Research Program of China (Grant No. 2006CB921101), NSFC Project for Excellent Research Team (Grant No. 60821004), and NSFC (Grant No. 60678029). M. X. acknowledges the funding support from NSF (US).

[1] P. Kumar and J. H. Shapiro, Opt. Lett. 10, 226 (1985).

[2] D. Grandclement, G. Grynberg, M. Pinard, Phys. Rev. Lett. 59, 44 (1987).

[3] J. Donoghue, M. Cronin-Golomb, J. S. Kane, and P. R. Hemmer, Opt. Lett. 16, 1313 (1991).

[4] M. Poelker, P. Kumar, and S.-T. Ho, Opt. Lett. 16, 1853 (1991).

[5] M. Poelker and P. Kumar, Opt. Lett. 17, 399 (1992).

[6] P. R. Hemmer, D. P. Katz, J. Donoghue, M. CroninGolomb, M. S. Shahriar, and P. Kumar, Opt. Lett. 20, $982(1995)$

[7] J. Okuma, N. Hayashi, A. Fujisawa, M. Mitsunaga, and K. Harada, Opt. Lett. 34, 698 (2009).

[8] H. Wu, M. Xiao and J. Gea-Banacloche, Phys. Rev. A 78, 041802(R) (2008).

[9] R. Guzman, J. C. Retamal, E. Solano, and N. Zagury, Phys. Rev. Lett. 96, 010502 (2006).

[10] H. Xiong, M. O. Scully, and M. S. Zubairy, Phys. Rev. Lett. 94, 023601 (2005). 
[11] X. Yu, D. Xiong, H. Chen, P. Wang, M. Xiao, J. Zhang, Phys. Rev. A 79, 061803(R) (2009).

[12] T. Debuisschert, A. Sizmann, E. Giacobino, and C. Fabre, J. Opt. Soc. Am. B 10, 1668 (1993).

[13] A. S. Villar, L. S. Cruz, K. N. Cassemiro, M. Martinelli, and P. Nussenzveig, Phys. Rev. Lett. 95, 243603 (2005).

[14] A. S. Villar, M. Martinelli, C. Fabre, and P. Nussenzveig, Phys. Rev. Lett. 97, 140504 (2006).

[15] A. S. Villar, K. N. Cassemiro, K. Dechoum, A. Z. Khoury, M. Martinelli, and P. Nussenzveig, J. Opt. Soc. Am. B 24, 249 (2007).

[16] A. S. Coelho, F. A. S. Barbosa, K. N. Cassemiro, A. S. Villar, M. Martinelli, P. Nussenzveig, Science 326, 823
(2009).

[17] V. D'Auria, S. Fornaro, A. Porzio, E. A. Sete, S. Solimeno, Appl. Phys. B 91, 309 (2008).

[18] X. Su, A. Tan, X. J. Jia, Q. Pan, C. Xie, and K. Peng, Opt. Lett. 31, 1133 (2006).

[19] J. Laurat, L. Longchambon, C. Fabre, and T. Coudreau, Opt. Lett. 30, 1177 (2005).

[20] C. F. McCormick, V. Boyer, E. Arimondo, and P. D. Lett, Opt. Lett. 32, 178 (2007).

[21] Quantum Information with Continuous Variables of Atoms and Light, edited by N. Cerf, G. Leuchs, and E. S. Polzik (Imperial College Press, London, 2007). 